J. Clin. Chem. Clin. Biochem.

Vol. 18, 1980, pp. 339-344

\title{
Eine gaschromatographische Methode zur gleichzeitigen Bestimmung von Ethosuximid und Valproinat im Serum
}

\author{
Von W. R. Külpmann \\ Institut für Klinische Chemie (geschäftsf. Direktor Prof. Dr. Dr. J. Büttner) Medizinische Hochschule Hannover
}

(Eingegangen am 14. November 1979/14. Februar 1980)

Zusammenfassung: Es wird ein gaschromatographisches Verfahren zur gleichzeitigen Bestimmung von Ethosuximid und Valproinat beschrieben. Nach Zusatz von inneren Standards wird die Serumprobe mit Chloroform und Schwefelsäure versetzt und extrahiert. $2 \mu \mathrm{l}$ der organischen Phase werden unmittelbar in den Gaschromatographen injiziert. Die Auftrennung erfolgt an SP 1000 isotherm, ohne Derivatbildung. Der Variationskoeffizient als Maß für die Präzision von Tag zu Tag betrug für Ethosuximid 2,6\% und für Valproinat 5,2\%. Bei Ringversuchen lagen die Meßwerte stets um weniger als eine Standardabweichung vom Sollwert entfernt. Die Nachweisgrenze lag mit 11,6 $\mu \mathrm{mol} / \mathrm{l}$ für Ethosuximid und 2,3 $\mu \mathrm{mol} / 1$ für Valproinat weit unterhalb des therapeutischen Bereichs. Zur Prüfung der Spezifität wurden u. a. die relativen Retentionszeiten von etwa 100 Arzneimitteln mit denen der Antiepileptica und ihren inneren Standards verglichen.

\section{A gaschromatographic method for the simultaneous determination of ethosuximide and valproate in serum}

Summary: A gaschromatographic procedure for the simultaneous determination of ethosuximide and valproate is presented. After addition of the two internal standards the serum sample is acidified and extracted by chloroform. $2 \mu \mathrm{l}$ of the organic phase is directly injected into the gaschromatograph without previous evaporation. The gaschromatographic analysis is carried out on SP 1000 under isothermal conditions without derivatisation. The coefficients of variation for the precision from day to day were $2.6 \%$ (ethosuximide) and $5.2 \%$ (valproate). In external quality control the results for both substances always differed less than one standard deviation from the mean. The detection limits $(11.6 \mu \mathrm{mol} / 1$ for ethosuximide and $2.3 \mu \mathrm{mol} / 1$ for valproate) were far below the usual therapeutic range. The specificity of the method was proven by comparison of the relative retention times of about 100 drugs, the investigated antiepileptic drugs and their internal standards.

\section{Einleitung}

Die Bedeutung der Úberwachung der Antiepilepticatherapie mit Hilfe von Konzentrationsbestimmungen im Blut unter definierten Entnahmebedingungen ist allgemein anerkannt $(1,2)$. Die Analysen werden zu einem großen Teil unter Einsatz der Gaschromatographie durchgeführt. Das vergleichsweise spät in die Therapie eingeführte Valproinat wird üblicherweise einzeln bestimmt (3-12), während man gewöhnlich die Ethosuximidkonzentration gemeinsam mit der der anderen Antikonvulsiva zu ermitteln versucht (13-21). Auf Grund des unter schiedlichen gaschromatographischen Verhaltens ist jedoch die Messung von Ethosuximid zusammen mit Carbamazepin, Phenobarbital, Phenytoin und Primidon unbefriedigend und führt dazu, daß das vergleichsweise weniger oft eingesetzte Ethosuximid die Optimierung der Verfahren für die häufiger applizierten Antiepileptica verhindert: Bei der Probenaufarbeitung muß die Flüchtigkeit des Ethosuximid be- rücksichtigt werden $(14,19)$ und bei der Gaschromatographie müssen Temperaturprogramme eingesetzt werden, die $100^{\circ} \mathrm{C}$ und mehr überbrücken müssen (14, $16,18,21)$.

Wir haben das seinerzeit von Vree et al. (5) ohne nähere Prüfung der Zuverlässigkeit für die Valproinatbestimmung vorgeschlagene Verfahren aufgegriffen und für die gemeinsame Bestimmung der in vielen Eigenschaften ähnlichen Verbindungen Valproinat und Ethosuximid modifiziert und evaluiert. Die Methode erlaubt nach gemeinsamer, einfacher Probenvorbereitung, die der leichten Flüchtigkeit der beiden Verbindungen Rechnung trägt, die gaschromatographische Bestimmung simultan, isotherm und ohne Derivatbildung durchzuführen. Damit ist die Möglichkeit gegeben, ein weiteres Verfahren zur Bestimmung der übrigen therapeutisch wichtigen Antiepileptica auf Carbamazepin, Phenobarbital, Phenytoin und Primidon zu beschränken und für diese gaschromato- 
graphisch vergleichsweise ähnlichen Substanzen die Bedingungen zu optimieren (22).

\section{Material und Methodik}

\section{Material}

Natriumvalproinat (2-Propyl-valeriansäure, Natriumsalz), $M_{\mathbf{r}}$ 166,2 (Labaz GmbH, Erkrath-Unterfeldhaus); Ethosuximid. (2-Ethyl-2-methyl-succinimid), $M_{\mathrm{r}}$ 141,2 (Parke, Davis \& Comp., München 2); Caprylsäure, $M_{\mathrm{r}} 144,2$ (Fluka Feinchemikalien $\mathrm{GmbH}, \mathrm{Neu}-\mathrm{Ulm}) ; 2$ 2,2,3-Trimethylsuccinimid, $M_{\mathrm{r}} 141,2$ (Aldrich-Europe, Nettetal 2). Schwefelsäure (6 mol/1), Natronlauge $(2 \mathrm{~mol} / \mathrm{l})$ und Eisessig, jeweils p. a. werden ohne Vorreinigung verwendet, Chloroform und Ethylacetat (p. a.) werden vor Gebrauch destilliert (Merck, Darmstadt). Ortho Anticonvulsant Control Serum (Ortho Diagnostics, Heidelberg). EMIT-aed ${ }^{\circledR}$ (Enzym-Immuno-Assay für die Ethosuximidbestimmung, Merck, Darmstadt).

\section{Lösungen}

Natriumvalproinat, Ethosuximid und 2,2,3-Trimethylsuccinimid werden in Ethylacetat-Eisessig $(100 \mathrm{ml}+1 \mathrm{ml}) \mathrm{bzw}$. in destilliertem Wasser gelöst: Konzentration: 50 bzw. $100 \mathrm{mg} / \mathrm{l}$. Haltbarkeit bei Raumtemperatur: Mindestens 3 Monate. Caprylsäure $(20 \mu \mathrm{l})$ wird in End-zu-End kalibrierten Kapillaren (Brand, Wertheim) aufgenommen und in $10 \mathrm{ml}$ Ethylacetat/Eisessig $(100 \mathrm{ml}+1 \mathrm{ml})$ ausgeschüttelt. Zur Herstellung der wäßrigen Caprylsäurelösung wird die gefüllte Kapillare $(20 \mu \mathrm{l})$ in $10 \mathrm{ml}$ Natronlauge $(0,1 \mathrm{ml} \mathrm{NaOH}(2 \mathrm{~mol} / \mathrm{l})+10 \mathrm{ml}$ dest. Wasser $)$ ausgeschüttelt. Haltbarkeit bei Raumtemperatur: Mindestens 3 Monate.

\section{Glasgeräte}

Spitzröhrchen mit Schliffstopfen, Inhalt $10 \mathrm{ml}$.

\section{Gaschromatographie}

Glassäule, silikonisiert, 1,2 m lang, Innendurchmesser $2 \mathrm{~mm}$. Säulenfüllung: 1\% SP 1000 auf Supelcoport, 100/120 mesh (Supelco, Bellefonte, USA). Gaschromatograph: Varian 1400 mit Flammenionisationsdetektor (Varian, Darmstadt). Säulentemperatur: $125^{\circ} \mathrm{C}$, Einlaßtemperatur: $180^{\circ} \mathrm{C}$, Detektortemperatur: $180^{\circ} \mathrm{C}$. Trägergas: Nachgereinigter Stickstoff $(40 \mathrm{ml} /$ $\min$ ).

\section{Me thodik}

$500 \mu \mathrm{l}$ des Patientenserums werden mit $50 \mu \mathrm{l}$ der wäßrigen Caprylsäurelösung (interner Standard für die Valproinatbestimmung) bzw. mit $50 \mu l$ der wäßrigen 2,2,3-TrimethylsuccinimidLösung (interner Standard für die Ethosuximidbestimmung) versetzt. $\mathrm{N}$ a ch Zugabe von $500 \mu \mathrm{l}$ Chloroform werden $50 \mu \mathrm{l}$ Schwefelsäure (6 mol/1) zugesetzt und 2 Minuten geschüttelt. Es wird 10 Minuten bei $3000 \mathrm{~g}$ zentrifugiert. $2 \mu \mathrm{l}$ der organischen Unterphase werden in den Gaschromatographen eingespritzt. Die Auswertung der Gaschromatogramme erfolgt über die Ausmessung der Peakhöhen von Proben und Standards.

\section{Ergebnisse}

\section{Präzision}

Die Präzision in der Serie wurde mit Serum bestimmt, das mit Ethosuximid und Valproinat aufgestockt wurde. Jeweils $500 \mu l$ wurden mit den inneren Standards versetzt und analysiert. In Tabelle 1 wurden die Ergebnisse zusammengefaßt. Die Variationskoeffizienten schwankten zwischen 1,0 und 2,2\%. Die Präzision von Tag zu Tag wurde mit einem ebenfalls selbst hergestellten, aufgestockten Serumpool und einem Kontrollserum (Ortho Anticonvulsant Control Serum) ermittelt. Dịe Variationskoeffiżienten betrugen zwischen 2,0 ünd 5,2\% (Tảb. 2).

\section{Richtigkeit und Spezifität}

Die Wiederfindung von Ethosuximid ohne Berücksichtigung des inneren Standards betrug $68,1 \%$ (Variationskoeffizient: 7,4\%, Anzahl $n=50$ ), die Wiederfindung von Valproinat wurde unkorrigiert $\mathrm{zu} 75,9 \%$ (Variationskoeffizient: $6,3 \%$, Anzahl $n=50$ ) bestimmt.

Die Gaschromatogramme von Pharmaka-freien Sera bzw. von Patienten unter Therapie mit Antikonvulsiva lassen keine Verunreinigungen oder störende Peaks von Metaboliten erkennen (Abb. 1-3).

Tab. 1. Gaschromatographische Bestimmung von Ethosuximid und Valproinat. Präzịsion in der Serie.

\begin{tabular}{llllll}
\hline Substanz & $\begin{array}{l}\text { Anzahl } \\
\text { der Analysen } \\
n\end{array}$ & $\begin{array}{l}\text { Miitelwert } \\
\mathrm{x}\end{array}$ & $\begin{array}{l}\text { Variations- } \\
\text { koeffizient } \\
(\mu \mathrm{mol} / \mathrm{l})\end{array}$ & $\begin{array}{l}\text { Sollwert } \\
\mu\end{array}$ & $\begin{array}{l}\overline{\mathrm{x}}-\mu \\
(\mu \mathrm{mol} / \mathrm{l})\end{array}$ \\
\hline Ethosuximid & 10 & 340,2 & 1,6 & 354,1 & $-3,9$ \\
& 10 & 677,0 & 1,0 & 708,2 & $-4,4$ \\
Valproinat & 10 & 285,8 & 1,2 & 300,8 & $-5,0$ \\
& 10 & 603,5 & 2,2 & $601, \overline{7}$ & $+0,3$ \\
\hline
\end{tabular}

Tab. 2. Gaschromatographische Bestimmung von Ethosuximid und Valproinat. Präzision von Tag zu Tag (Einzelwerte).

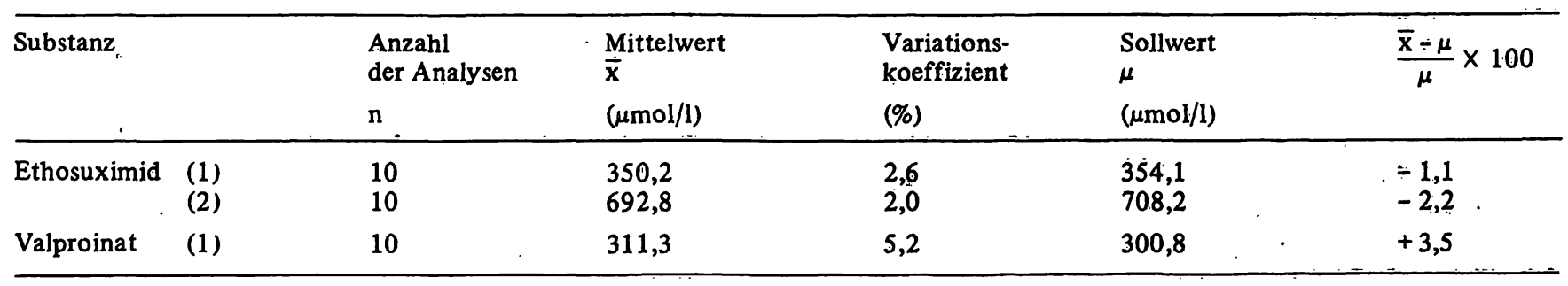

(1) Selbst hergestelltes, aufgestocktes Poolserum

(2) Ortho Anticonvulsant Control Serum 


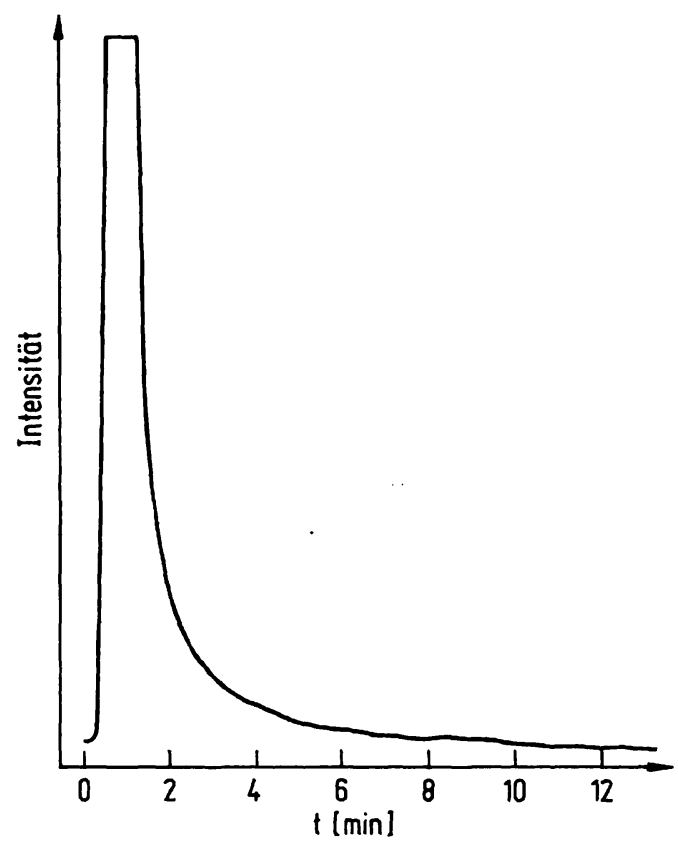

Abb. 1. Gaschromatogramm eines medikamentenfreien Serumextraktes.

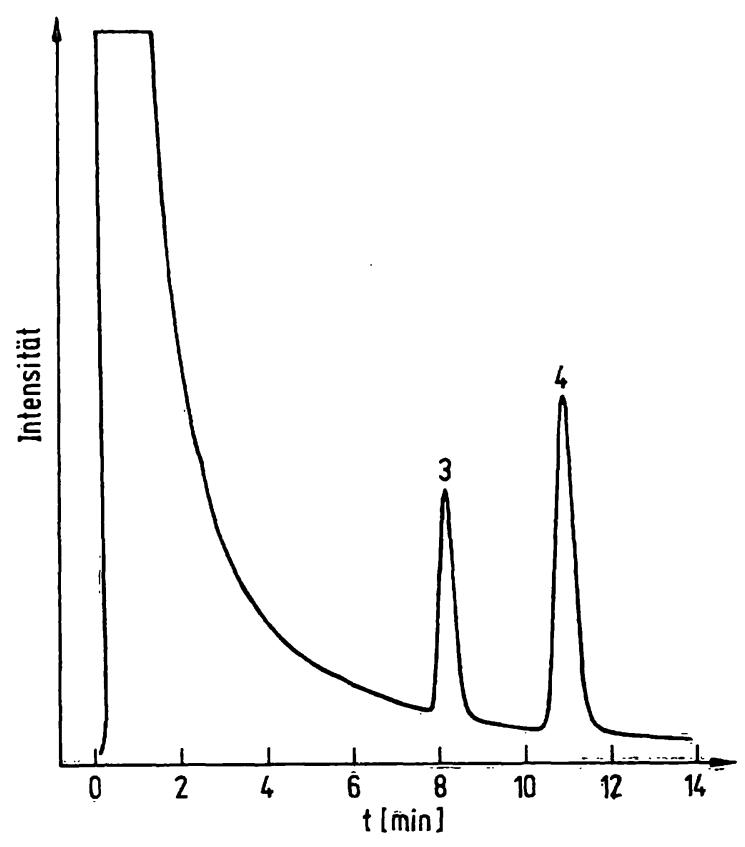

Abb. 3. Gaschromátogramm eines Serumextraktes von einem Patienten unter Erhosuximid therapie.

3: 2,2,3=Trime thylsuccinimid (innerer Standard)

4: Ethosuximid $(747 \mu \mathrm{mol} / \mathrm{l})$.

Es wurde gep̣rüft, ob die folgenden Medikamente auf Grund ihrer Retentionszeit und ihres Extraktionsverhaltens störren könnten. Dazu wurden jeweils $10 \mathrm{mg}$ der Reinsubstand bzw. $5 \mathrm{ml}$ der Flüssigkeit verwêndet, die in $10 \mathrm{ml}$ der organischen Unterphase einer Mischung von Chloroform und Schwefelsäure $(6 \mathrm{~mol} / \mathrm{l})(100 \mathrm{ml}+$ $10 \mathrm{ml}$ ) gelöst wurden. $2 \mu \mathrm{l}$ wurden in den Gaschromatographen injiziert. Auf diese Weișe wird zugleich die Lös-

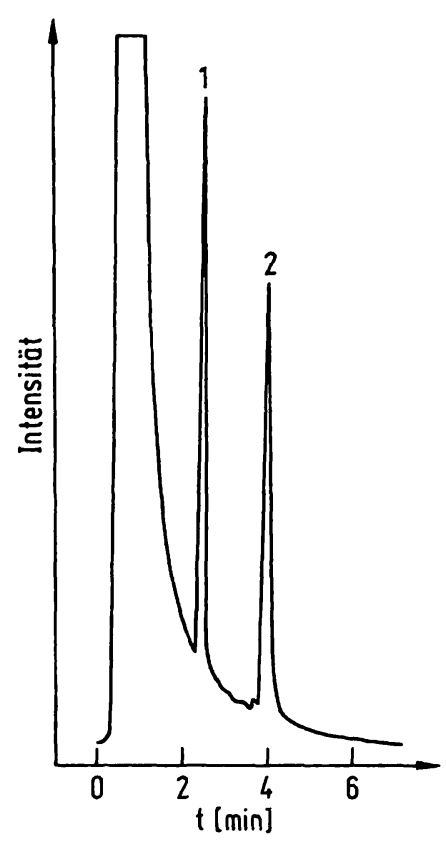

Abb. 2. Gaschromatogramm eines Serumextraktes von einem Patienten unter Valproinattherapie.

1: Valproinat (503 $\mu \mathrm{mol} / \mathrm{l})$

2: Caprylsäure (innerer Standard)

lichkeit als auch das gaschromatographische Verhalten der Substanzen geprüft, und eine mögliche Störung der Analyse durch ein Pharmakon in einem Schritt erfaßt. Andererseits sind deshalb genaue Angaben der geprüften Konzentrationen nicht zu geben. Auf Grund der eingesetzten Mengen liegen sie jedoch im toxischen und letalen Bereich.

Die Ethosuximidbestimmung wird durch keines der aufgeführten Medikamente gestört:

Acidum acetylosalicylicum

Acidum ascorbicum

Acidum niflumicum

Adipiodon (Methylglucamins. 5. ml)

Allopurinol

Amidotrizoesäure (Methylglucamins. $5 \mathrm{ml}$ )

Aminophenazon

Amitryptilin

Ampicillin

Amphetamin

Antażolin

Azapropazon

Benzbromaron

Bisacodyl

Carbamazepin

Carbocromen

Carbromal

Cetobemidon

Chlordiazepoxid

Chloroquin

Chlorprothixen

Clofibrat 
Clomethiazol

Clonazepam

Codein

Cyclophosphamid

Dextran (5 ml)

Dextromoramid

2,2-Diethylallylacetamid

Diazepam

Digoxin

Diphenhydramin

Doxepin

Ethinamat

Fenetyllin

Fenfluramin

Fluphenazin

Furosemid

Gentamycin

Glafenin

Glibenclamid

Glutethimid

Haloperidol

Hyoscin-N-butylbromid

Imipramin

Indometazin

Levorphanol

Mebhydrolin

Meclizin

Mephenytoin

Mesuximid

Methadon

Methaqualon

Methotrexat

Methyldopa

Methylpentynol

Methylstiryldibromhydantoin

Methyprylon

Mianserin

Miroton ${ }^{\circledR}$

Modenol $^{\circledR}$

Morphin

Neoplasmagel $(5 \mathrm{ml})$

Nicotinamid

Nitrazepam

Nitrofurantoin

Nomifensin

Noramidopyrini methanosulfonas natrium

Norfenefrin

Normethadon

Oxazepam

Oxyphenbutazon

Paramethadion

Paraxin $^{\circledR}$

Penicillamin

Phenylethylmalondiamid
Phenformin

Pheniramin- $p$-amino-salicylat

Phenobarbital

Phenprocumon

Phenylbutazon

Phenytoin

Polybion ${ }^{\circledR}$

Prazepam

Prednisolon

Primidon

Probenecid

Promethazin

Prothipendyl

Pyrithyldion

Reserpin

Spironolacton

Sulfadiazin

Sulfametoxydiazin

Sultiam

Tetracyclin

Thioridażin

Tolbutamid

Triflupromazin

Trimethadion

Die Bestimmung von Valproinat wird lediglich durch hohe Konzentrationen von Carbromal und Clomethiazol gestört. Beim Vergleich der Ërgebnisse, die durch Analyse von Ethosuximidhaltigen Sera mittels eines homogenen Enzymimmunoassays (EMIT ${ }^{\circledR}$ ) und gaschromatographisch erhalten wurden, ergab sich eine gute UUbereinstimmung (23). Die Abweichungen, die bei Ringversuchen beobachtet wurden (Ringversuchsleitung: Dr. A. Richens, St. Bartholomew's Hospital Quality Control Scheme for Antiepileptic Drugs, London), lagen bei den gaschromatographischen Analysen immer um weniger als eine Standardabweichung vom Mittelwert entfernt (Tab. 3).

\section{Empfindlichkeit}

Die Empfindlichkeit der Methode wurde abgeschätzt durch Bestimmung der dreifachen Standardabweichung bei Analysen im unteren Konzentrationsbereich als einem Maß für die Präzision bei der Konzentration $0 \mu \mathrm{mol} / \mathrm{l}(24)$. Eine Bestimmung des mittlèren Leerwertes ist nicht möglich, zumal die Retentionszeit im untersten Konzentrationsbereich nicht konstant ist. Als Abschätzung kann der Vergleich zwischen Meß- und Sollwert dienen (Tab. 4). Jedenfalls erlaubt die Methode auch Konzentrationen unterhalb des therapeutischen Bereich (Ëthosuximid: 424,8-566,4 $\mu \mathrm{mol} / 1$ und Valproinat: $349,0-698,0 \mu \mathrm{mol} / 1$ (25)) zuverlässig zu bestimmen. .

\section{Praktikabilität}

Diè Probenvorbereitung erfordert für 10 Analysen höchstens eine halbe Stunde. Alle 6 Minuiten kànn ěín Vàl- 
Tab. 3. Gaschromatographische Bestimmung von Ethosuximid und Valproinat. Externe Qualitätskontrolle.

\begin{tabular}{|c|c|c|c|c|c|}
\hline Substanz & $\begin{array}{l}\text { Anzahl } \\
\text { der Teilnehmer } \\
\mathrm{n}\end{array}$ & $\begin{array}{l}\text { Mittelwert } \\
(\mu \mathrm{mol} / \mathrm{l})\end{array}$ & $\begin{array}{l}\text { Variationskoeffizient } \\
\text { von } \bar{y} \\
(\%)\end{array}$ & $\begin{array}{l}\text { Eigener Meßwert } \\
x \\
(\mu \mathrm{mol} / 1)\end{array}$ & $\frac{x-\bar{y}}{\bar{y}} \cdot 100$ \\
\hline Ethosuximid* & $\begin{array}{l}74 \\
76 \\
78 \\
69 \\
74\end{array}$ & $\begin{array}{l}407,1 \\
207,2 \\
109,9 \\
200,2 \\
220,6\end{array}$ & $\begin{array}{l}14,5 \\
20,7 \\
27,4 \\
24,3 \\
19,6\end{array}$ & $\begin{array}{r}400,6 \\
230,1 \\
96,5 \\
190,5 \\
224,4\end{array}$ & $\begin{array}{l}-1,6 \\
+11,1 \\
-13,9 \\
-\quad 4,9 \\
+\quad 1,7\end{array}$ \\
\hline Valproinat* & $\begin{array}{l}44 \\
55 \\
65 \\
82 \\
86 \\
87 \\
83 \\
94\end{array}$ & $\begin{array}{l}298,0 \\
329,0 \\
347,5 \\
105,1 \\
452,3 \\
257,6 \\
644,5 \\
188,6\end{array}$ & $\begin{array}{l}13,4 \\
11,9 \\
17,3 \\
20,7 \\
15,6 \\
15,1 \\
18,5 \\
18,7\end{array}$ & $\begin{array}{l}300,7 \\
332,2 \\
380,2 \\
110,6 \\
411,1 \\
257,5 \\
617,5 \\
174,9\end{array}$ & $\begin{array}{r}+0,9 \\
+1,0 \\
+9,4 \\
+\quad 5,3 \\
-\quad 9,1 \\
0,0 \\
-\quad 4,1 \\
-\quad 7,3\end{array}$ \\
\hline
\end{tabular}

* bezogen auf gaschromatographische Verfahren ohne Derivatbildung

Tab. 4. Gaschromatographische Bestimmung von Ethosuximid und Valproinat. 3 s-Bereich bei niedriger Konzentration.

\begin{tabular}{|c|c|c|c|c|c|}
\hline \multirow[t]{2}{*}{ Substanz } & $\begin{array}{l}\text { Anzahl } \\
\text { der Analysen }\end{array}$ & $\begin{array}{l}\text { Sollwert } \\
\mu\end{array}$ & $\frac{\text { Mittelwert }}{\bar{x}}$ & $3 \mathrm{~s}$ Bereich & $\begin{array}{l}\text { Therapeu- } \\
\text { tischer Bereich } \\
\text { nach I. c. ( } 25)\end{array}$ \\
\hline & $\mathrm{n}$ & $(\mu \mathrm{mol} / 1)$ & $(\mu \mathrm{mol} / \mathrm{l})$ & $(\mu \mathrm{mol} / \mathrm{l})$ & $(\mu \mathrm{mol} / \mathrm{l})^{\prime}$ \\
\hline $\begin{array}{l}\text { Ethosuximid } \\
\text { Valproinat }\end{array}$ & $\begin{array}{l}10 \\
10\end{array}$ & $\begin{array}{l}35,4 \\
30,1\end{array}$ & $\begin{array}{l}34,7 \\
35,0\end{array}$ & $\begin{array}{r}11,6 \\
2,3\end{array}$ & $\begin{array}{l}424,8-566,4 \\
349,0-698,0\end{array}$ \\
\hline
\end{tabular}

proinathal tiger Extrakt zur Analyse eingespritzt werden, alle 13 Minuten ein Ethosuximidhaltiger. Eine Störung durch Verunreinigungen mit langen Retentionszeiten, welche die Auswertung der anschließenden Gaschromatogramme erschweren, wurde nicht beobachtet. Die Materialkosten sind minimal, was einen wesentlichen Vorteil gegenüber einer entsprechenden EMIT ${ }^{\circledR}$-Analyse darstellt, zumal bei dem vergleichsweise weniger oft eingesetzten Ethösuximid die besonders teuren Einzelanalysen (26) häufig sind.

\section{Diskussion}

Im Gegensatz zu anderen gaschromatographischen Methoden werden Ethosuximid und Valproinat gleichzeitig bestimmt. Dieses Vorgehen empfiehlt sich auf Grund der ähnlichen Substanzeigenschaften, die diese Antikonvulsiva von den anderen Antiepileptica wesentlich abheben. Die Flüchtigkeit der Verbindungen zusammen mit ihren hohen therapeutischen Konzentrationen im Serum erfordert und erlaubt eine Extraktion ohne anschließende Konzentrierung des Extraktes, z. B. durch Eindampfen. Die gaschromatographische Analyse kann simultan, isotherm und ohne Derivatbildung durchgeführt werden, was die bei den ständig̈ steigenden Analysenanforderungen dringliche Mechanisierung der gaschromatographischen Bestimmung erleichtert.

\section{Danksagung}

Frau L. Lütke-Holz und Herrn K. Petry danke ich für die zuverlässige Mitarbeit bei der Durchfụhrung der Bestimmungen.

\section{Literätur}

1. Lund, L. (1974), Arch. Neurol. 31, 289-294.

2. Schmidt, D. (1977), Therapiewoche 27, 501-510.

3. Meijer, J. W. A. \& Hessing-Brand, L. (1973), Clin. Chim.

Acta 43, 215-222.

4. Loiseau, P., Brachet, A. \& Henry, P. (1975), Epilepsia 16, 609-615.

5. Vree, T. B., van der Kleijn, E. \& Knop, H. J. (1976), J. Chromatogr. 121, 150-152.

6. Dacremont, G. \& Cocquyt, G. (1977), Acta Paediatr. Belg. $30,41-44$.

7. Dusci, L. J. \& Hackett, L. P. (1977), J. Chromatogr. 132, 145-147.

8. Fellenberg, A. J. \& Pollard, A. C. (1977), Clin. Chim. Acta 81, 203-208.

9. Jensen, C. J. \& Gugler, R. (1977), J. Chromatogr. 137, 188-193. 
10. Löscher, W. (1977), Epilepsia 18, 225-227.

11. Schulz, H.-U. \& Toseland, P. A. (1977), Ann. Clin. Biochem. $14,240-242$.

12. Wood, M. H., Sampson, D. C. \& Hensley, W. J. (1977), Clin. Chim. Acta 77, 343-347.

13. Cremers, H. M. H. G. \& Verheesen, P. E. (1973), Clin. Chim: Acta 48, 413-420.

14. Beam, R. E. (1974), Am. J. Med. Technol. 40, 211-218.

15. Perchalski, R. J. \& Wilder, B. J. (1974), J. Pharm. Sci. 63, 806-807.

16. Toseland, P. A., Albani, M. \& Gauchel, F. D. (1975), Clin. Chem. 21, 98-103.

17. Latham, A. N. \& Varlow, G. (1976), Br. J. Clin. Pharmacol. $3,145-150$.

18. Abraham, C. V. \& Gresham, D. (1977), J. Chromatogr. 136, $332-336$.
19. Heipertz, R., Pili, H. \& Eickhoff, K. (1977), Clinn: Chim. Acta $77,307-316$.

20. Hill, R. E. \& Latham, A. N. (1977), J. Chromantoğr. 131, $341-346$.

21. Sengupta, A. \& Peat, M. A. (1977), J. Chromatogr. 137, 206-209.

22. Külpmann, W. R. (1979), J. Clin. Chem. Clis.. Biochem. 17, $174=175$.

23. Külpmañn, W. R. \& Oellerich, M. In Voorbereitung.

24. Büttner, J., Borth, R., Boutwell, J. H., Broughton, P. M. G. \& Bowyer, R. C. (1976), Clir. Chim. Acta 69, F1-F17; J. Clin. Chem. Clin. Biochem. 14, 265-275 (1976); Clin. Chem. 22, 1922-1932 (1976).

25. Klotz, U. (1978), Fortschr. Med. 96, 9-13.

26. Oellerich, M., Külpmann, W. R., Haeckel, R. \& Heyer, R. (1977), J. Clin. Chem. Clin. Biochem. 15, 353-358.

Priv.-Doz. Dr. W. R. Külpmann Institüt für Klinische Chemie Karl-Wiechert-Allee 9. 3000 Hannover 61 